\title{
Expected Reliability of Everyday- and Ambient Assisted Living Technologies -
}

\author{
Results From an Online Survey
}

\author{
Frederick Steinke \\ Humboldt-Universität zu Berlin \\ Berlin, Germany \\ Tobias Fritsch \\ Universität Heidelberg \\ Heidelberg, Germany
}

\author{
Andreas Hertzer \\ Universität Augsburg \\ Augsburg, Germany \\ Helmut Tautz \\ Technische Hochschule Ingolstadt \\ Ingolstadt, Germany
}

\author{
Simon Zickwolf \\ Ludwig-Maximilians-Universität München \\ Munich, Germany
}

\begin{abstract}
To receive valuable information about expected reliability in everyday technologies compared to Ambient Assisted Living (AAL) technologies, an online survey was conducted including five everyday (train, dishwasher, navigation system, computer, mobile phone) and three AAL (stove, window, floor sensors) technologies. The age range of the 206 participants (109 men; 97 female) was from 14 to 88 years (mean=38.0). The descriptive analysis indicates expected reliabilities of more than $\mathbf{9 0 \%}$ for most technologies. Only train punctuality is considered as less reliable with a mean expected reliability of $86 \%$. Furthermore, by using t-tests it can be shown that the three AAL technologies are expected to have a higher reliability than the everyday technologies. Additionally, a sample split at the age of 50 years indicates that elderly participants expect that technologies have a higher reliability than younger participants do. Using these findings, in a next step an experiment with different reliability levels of AAL technologies will be designed. This differentiation will be used to measure the influence of reliability on trust and intention to use in context of Ambient Assisted Living.
\end{abstract}

Keywords-Ambient Assisted Living; Elderly People; Expected Reliability; Online Survey; Technology

\section{INTRODUCTION}

Elderly people are an interesting target group for companies to sell their products because of their rising percentage among the worldwide population [1]. Due to the fact, that this target group is often financial strong and has a higher income, there is more money left for consumption [2]. For this case, science deals for several decades with the research of new technologies to support people in their own home [3]. As a result, different concepts have entered the market [4][5][6][7][8]. On this basis, dependencies between different technologies and variables like age are a very important subject for research.
In consequences of the demographic change, the proportion of elderly people, who would like to spend an independent life at home, is increasing. The market for technology-supported systems for the use at home is growing because of the physical effects of older people [9]. Therefore, concepts like Ambient Assisted Living are getting more attractive in the last years. With the aid of sensors and actuators within the framework of an intelligent platform- the time older people can live independently can be extended. By the use of AAL it is possible to use pervasive devices for integrating them into a reliable environment for the elderly. Ambient Intelligence enables automatic services which are dependent on the need of the user and can be seen as essential part of AAL [10]. By means of summarizing and demand-oriented analyzing of sensor data, an individualization of care as well as nursing services is possible [5]. Product designers of such technologies have to consider a lot of different factors in the process of development to design a marketable solution. As one of these factors, the reliability of the technique in general is a crucial point [11][12].

For that reason the present study discusses the expected reliabilities for different technologies (including AAL) by the users. The following brief description should help to gain a better structural overview. The background section includes former studies which demonstrate the importance of knowing the expected reliability of users and brings it in context with AAL. In the next step, a description about the methodology for getting the required information by an online questionnaire and the sample details are presented. Afterwards, the results by means of a descriptive analysis, correlation analysis, t-tests, and analyses of variances are underlined. The correlation analysis shows the dependences between expected reliability of users on different technologies and other variables like age and gender. With the aid of paired-sample t-tests, the expectations about reliabilities of different technologies are shown. An 
Omitted Least Squares (OLS) regression illustrates expected reliability by AAL-related and everyday-technologies in connection with other variables (e.g., gender, age).

Finally, a conclusion section describes the participants' expectations between reliability of AAL and everyday technologies. Additionally, recommendations for the designing of AAL technologies are given. For this reason, the following research question is answered: What differences exist regarding the expected reliability of everyday vs. Ambient Assisted Living Technology? A brief review about the limitations concerning the sample and test execution as well as further research needs round off the survey.

\section{BACKGROUND}

Knowledge about the reliability of different technologies expected by the users is highly important as reliability is a crucial component of the technology adaption decision [13]. In order to address potential users more appropriately, a view on their expected reliability on different technologies is the object of this study. According to the Oxford Dictionary, reliability means the "consistently good in quality or performance" and therefore, the ability to be trusted [14]. Thus, it is very important for a product or service to meet the users' expected reliabilities to get their trust for using it.

"Expected", in this context, could be understood as fulfilling the personal requirements of each user about the system-functionality [15]. Consequently, expected reliability can be seen as a pre-condition for building user-trust [16]. Some former studies already described the relationship between reliability of technologies and their consequences in different ways. One example is a test which examined the influence of trust and etiquette in high-criticality automated systems. In this study, user performance was much better when the automation reliability was higher and good automation etiquette also contributed to a better performance [17]. AAL systems could be seen as high-criticality automated systems as well wherefore reliability of the system will be important. In another study [18] groups were divided into younger (age 2045 ) and older (60-80) adults. Nevertheless, it was also obvious that both groups will begin to appropriately use the systems if they work in a proper way. Especially older adults are willing to change their behavior when the system was useful and they can trust on their reliability [18].

In order to get the required information from the user, different types of sensors are in the field. Radio-frequency identification (RFID), motion detectors, heat, and pressure sensors for example are in use to send up information to the system for the purpose of doing the right actions at the right time. It is possible to switch off the oven if somebody had forgotten to do that or to do an emergency call after a person slumped on the floor because of a qualm. To realize these lifesaving measures it is very important to have a reliable interaction between sensor and actuator [19]. Furthermore, it is already possible to transmit physiological and psychological information about the user. With the help of sensors attached to the user's body and video cameras and microphones it is also manageable to get a pattern of respiration and features of facial expression [20]. A disadvantage for the user due to the physical and social discomfort by wearing such devices could be reduced by advances in miniaturization of the devices.

However, a study revealed that fixed attachment of sensors in the accommodation was considered to be more reliable than attachment to clothing or on/ in the body. In addition, reliability and ease of use were also assessed as highly important as a basis for trust in AAL technology [21].

To check how different technologies are evaluated, the present study is conducted to analyze differences between everyday used technology and AAL technology.

\section{METHODS}

To gather information about whether the expected reliability (ER) in a working system differs between technologies and between younger and older people, several scenarios from daily life are considered in an online questionnaire. To recruit participants, emails were sent out to students and to acquaintances of the authors requesting for participation and for forwarding the email (mainly to persons older than the age of 50).

The survey was conducted on a three-week period in January 2013 using the web page "oFb - der onlineFragebogen". The first part of the questionnaire contained 14 questions regarding eight scenarios.

Five scenarios were queried with one question each, dealing with the topics train punctuality, dishwasher functioning, navigation system functioning, computer functioning, and mobile phone functioning. These scenarios are considered as everyday technologies in the paper at hand. Participants had to decide on how reliable they believed the technologies worked. The reliability scale ranged from " $70 \%$ or less" to " $100 \%$ " in steps of 5\%. To answer the questions a 7-point Likert scale was used.

Following, three technical assistance scenarios in the context of AAL were examined, queried by three questions each. These scenarios are similar to those queried in [22] and dealing with AAL technologies as well as sensor devices. One scenario considered the possibility to turn off the stove via an application on a tablet computer. A second scenario considered a situation where sensor technology detects a person lying on the floor and automatically calls an ambulance. The third scenario again dealt with actively executing a computer application, but this time the application enables the person to open or close the windows via the Internet. Participants had to evaluate their expected reliability, i.e. how well the technological instruments described would perform. The reliability scale again was set from " $70 \%$ or less" to " $100 \%$ ".

The order of the scenario-based questions was the following: The different scenarios from everyday- and AAL technologies were queried in random order but the three questions of each AAL scenario were queried together.

Additionally to these reliability related questions, participants had to answer socio-demographic questions about their age, gender, and living condition as well as whether they possessed a smartphone, and about their computer, and tablet computer experience. 


\section{A Sample}

In the three-week period, 251 persons started the survey and 206 persons finished it. The following analysis will only consider individuals that finished the survey and will refer to them as "participants." 52.9 percent of the participants were men and 47.1 percent were women. The average age was 38.0 years $(\mathrm{SD}=17.0)$ and the median age was 29. The exact distribution in eight age-categories is shown in figure 1.

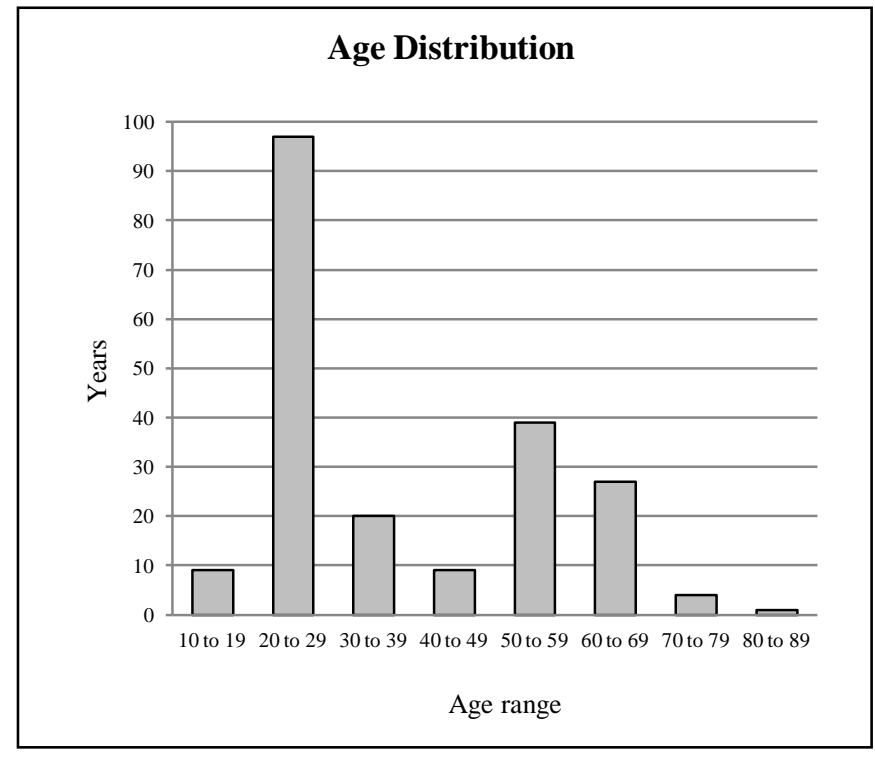

Fig. 1. Illustration of participant age distribution.

The reason for this age distribution is the fact that students were addressed and that they were asked to forward the mail to two persons who were older than 50.

27 percent of the participants were living alone $(\mathrm{m}=33 \%$, $\mathrm{f}=21 \%$ ) and 63 percent were in possession of a smartphone $(\mathrm{m}=67 \%, \mathrm{f}=60 \%)$. The average participant has been using PCs for 16.9 years and tablet PCs for 0.45 years. The average usage time of PCs is 37.4 hours per week, that of tablet PCs 1.8 hours per week. On the other hand, those people using tablet PCs, i.e. $24.8 \%$ of the sample, on average use it 7.1 hours per week.

The following table illustrates these descriptive variables while differentiating between male and female participants.

TABLE I. SAMPLE DESCRIPTIVES

\begin{tabular}{|l|l|l|l|}
\hline & Total Sample & Male & Female \\
\hline $\mathrm{N}$ & 206 & 109 & 97 \\
\hline Age & 37.97 & 35.38 & 40.35 \\
\hline Living alone & $27 \%$ & $33 \%$ & $21 \%$ \\
\hline Smartphone possession & $64 \%$ & $67 \%$ & $60 \%$ \\
\hline $\begin{array}{l}\text { Computer experience } \\
\text { (years) }\end{array}$ & 15.77 & 16.89 & 14.52 \\
\hline $\begin{array}{l}\text { Computer usage (hours } \\
\text { per week) }\end{array}$ & 37.40 & 41.50 & 32.80 \\
\hline $\begin{array}{l}\text { Tablet experience } \\
\text { (years) }\end{array}$ & 0.45 & 0.44 & 0.45 \\
\hline $\begin{array}{l}\text { Tablet usage (hours per } \\
\text { week) }\end{array}$ & 1.81 & 1.58 & 2.07 \\
\hline
\end{tabular}

\section{RESULTS}

This section will present the results from the online survey and will mainly focus on the differences across the technologies. First, a descriptive analysis is executed showing basic information about the results of the survey. Second, a correlation analysis is executed to indicate important relationships. Third, t-tests and analyses of variances are considered. Fourth, a regression section concludes the results section. Fifth, the sample is split into two groups dependent on the participants' age to analyze differences between older and younger participants.

\section{A Descriptive analysis}

The following graph illustrates the mean of expected reliabilities of the technologies.

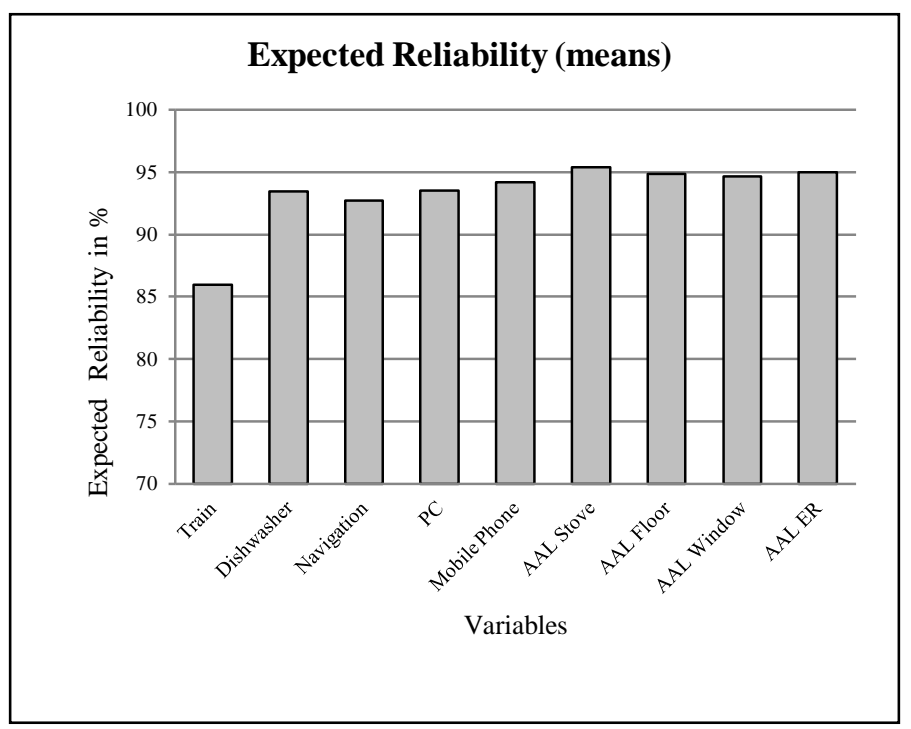

Fig. 2. Illustration of mean expected reliabilities.

As can be seen in the graph, all mean expected reliabilities exceed $85 \%$ or even $90 \%$. One technology (stove) even exceeds the $95 \%$ level of expected reliability. Furthermore, the graph indicates that the expected reliability of the train scenario is lower than the expected reliabilities of the other technologies and that the AAL-related technologies (stove, floor, and window, together summarized in the variable AAL ER) are expected to have a higher reliability than all other technologies. The mean expected reliabilities as well as their standard deviations are illustrated in Table II.

TABLE II. COMPARISON OF DIFFERENT TECHNOLOGIES REGARDING EXPECTED RELIABILITY

\begin{tabular}{|l|l|l|}
\hline Scenario & Mean & Standard Deviation \\
\hline Train & 0.860 & 0.094 \\
\hline Dishwasher & 0.934 & 0.057 \\
\hline Navigation System & 0.927 & 0.062 \\
\hline Computer & 0.935 & 0.053 \\
\hline Mobile Phone & 0.942 & 0.052 \\
\hline Stove & 0.953 & 0.052 \\
\hline Floor sensor & 0.949 & 0.062 \\
\hline Window & 0.947 & 0.057 \\
\hline $\begin{array}{l}\text { AAL technologies } \\
\text { (combined) }\end{array}$ & 0.949 & 0.048 \\
\hline
\end{tabular}




\section{B Correlation analysis}

The correlation analysis reveals several important aspects regarding the relationship between the different technologies. First, positive correlations exist between the expected reliabilities for all different technologies (correlations ranging from 0.211 to 0.690 ), meaning that participants who consider one technology to be reliable tend to consider another technology reliable as well.

With respect to the participant's gender no clear result can be drawn regarding the expectations of the technologies' reliabilities except for the window and stove technology, where being a woman is negatively correlated with expected reliability (significant correlations of -0.154 for the window and -0.159 for the stove technology).

The age of the participants, on the other hand, was positively correlated with the expected reliabilities. The corresponding correlations range from 0.178 to 0.366 , all significant on at least the 5\% level. This indicates that elderly participants expect the technologies to have a higher reliability than younger participants do.

The possession of a smartphone is negatively correlated with the expectation of reliability. Additionally, the number of years a participant has been using computers is positively correlated with his opinion of the reliability of the AAL technologies. Contrary to the other technologies (nonsignificant correlations of 0.019 to 0.102 ) the correlations with the technologies stove (0.272), floor (0.216), and window $(0.214)$ are significant at the $1 \%$ level.

On the other hand, neither living alone nor the variables regarding the tablet experience (in years), the weekly usage of computers, or that of tablet computers have clear relationships with expected reliability.

\section{$C$ Analysis using t-tests}

The analysis of the means using a one-sample t-tests as well as paired sample t-tests showed significant differences in the perception of the different technologies. The mean expected reliabilities are significantly greater than $80 \%$ but differ across technologies.

The one-sample t-tests show that while the train scenario is expected to have the lowest reliability, i.e. significantly lower than $90 \%$ but higher than $80 \%$, all other technologies show expected reliabilities significantly higher than $90 \%$.

The paired-sample t-tests again indicate that the reliability of the punctuality of trains is significantly lower than that of all other technologies $(p<0.001$ for all technologies). The reliabilities of the AAL technologies, on the other hand, are expected to be the most reliable technologies. The respective expected reliabilities of the stove, window, and floor technologies are significantly higher than those of the train, dishwasher, navigation system, and PC technologies and nonsignificantly higher than the expected reliability of the mobile phone technology.

\section{$D$ Regression analyses}

An OLS regression with the mean of all AAL-related expected reliabilities as the dependent and the non-AAL- related technologies (train, dishwasher, navigation system, PC, mobile phone) as well as gender, age, living environment, and smartphone possession indicates the following: The expected reliabilities of the train $(\mathrm{p}<0.05)$ and navigation system $(p<0.01)$ technologies as well as the age of the participant $(p<0.05)$ and being male $(p<0.005)$ have a significant positive effect on the expected reliability of the AAL technologies, the other variables are not influential.

\section{E Sample Split at age of 50}

To analyze possible differences between younger and older participants, a split is executed at the age of 50. The group of "younger" persons (below the age of 50) consisted of 135 participants and the group of "older" persons (at least of age 50) of 71 participants. The following figure shows the mean expected reliabilities differentiated between younger and older participants.

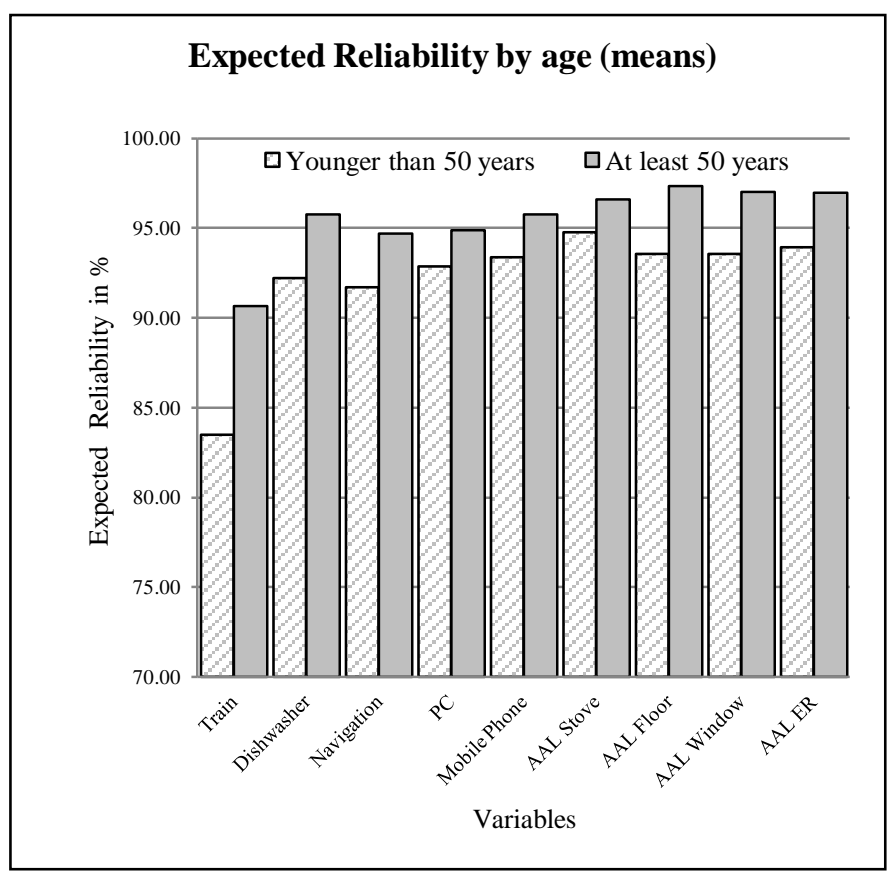

Fig. 3. Comparison of younger and older participants.

The graph shows that younger participants of the survey on average reported lower expected reliabilities than older participants for all technologies. In the train scenario, younger participants expected the punctuality to be less than $85 \%$ whereas older participants reported an average value of more than $90 \%$. In the other everyday technologies as well as in the AAL scenarios younger participants reported average values between 90 and $95 \%$ while participants of at least 50 years of age reported average values of close to or even exceeding $95 \%$ reliability.

Sample t-tests comparing all reliability variables show significant differences for all expected reliabilities. They also show that the two groups differ with respect to mobile phone possession, computer experience, and computer usage per week. The following table shows the mean differences between younger and older participants with respect to expected reliability. 
TABLE III. MEAN DIFFERENCES OLDER VS. YOUNGER

\begin{tabular}{|l|l|l|}
\hline Variable & Mean Difference* & Significance \\
\hline Train & 0.071 & $<0.001$ \\
\hline Dishwasher & 0.036 & $<0.001$ \\
\hline Navigation system & 0.030 & $<0.005$ \\
\hline Computer & 0.020 & $<0.01$ \\
\hline Mobile phone & 0.024 & $<0.005$ \\
\hline Stove & 0.018 & $<0.05$ \\
\hline Floor sensor & 0.037 & $<0.001$ \\
\hline Window technologies & 0.035 & $<0.001$ \\
\hline $\begin{array}{l}\text { AAL } \\
\text { (combined) }\end{array}$ & 0.028 & $<0.001$ \\
\hline
\end{tabular}

* Represents the difference between older and younger participants

\section{DISCUSSION}

The study describes the differences of expected reliabilities between everyday- and AAL technologies in dependency of user-age to gain deeper insights about differences between young and older users (cut-off age: 50 years) regarding the expected reliabilities of the technologies considered.

The results of the study show that the participants of the study expected all reliabilities of the technologies to be far from the minimum value $(70 \%)$ but often close to the maximum value $(100 \%)$. This indicates high expectations with respect to the technologies queried in the survey. Furthermore, the results indicate differences in expected reliability among different technologies as well as among different groups of age.

As mentioned above, two important aspects exist with respect to the differentiation between technologies. First, the expected reliability of trains is evaluated significantly lower than that of all other technologies. That points to negative sentiments of participants towards the German railway system. Second, the scenarios regarding AAL technologies were evaluated as being more reliable than the everyday technologies. This shows high expectations of people towards such high-criticality systems compared to technologies which people are used to, such as mobile phones and dishwashers.

The study conducted analyzed the expected reliabilities of five everyday technologies, as well as three AAL-related technologies. The results indicate a reliability expectancy of more than $90 \%$ for all technologies except for the train scenario. The reliability of train schedules (i.e. train punctuality) was expected to be close to $86 \%$. The comparison between these two types of technologies revealed significant differences with respect to the expected reliabilities. AAL technologies are expected to show a higher reliability than the everyday technologies.

The comparisons between younger and older participants further revealed that older persons, i.e. persons of at least 50 years of age, expect all technologies to be more reliable than younger persons do. In connection with AAL technology, the age of the participants as well as the gender male shows a significant positive effect on the expected reliability. This is an important result considering the target group of AAL products, namely elderly people. For producers of AAL technologies it is very important to know about the expectations elderly persons have regarding the reliability of high-criticality systems.

\section{CONCLUSION}

The central statements of this study regarding expected reliability of different technologies subject to the age of the participants should be taken into account for providers of AAL technologies. The results already treated in the discussion section give interesting insights which should be considered for addressing the target group for AAL products. The combination of the findings leads to the conclusion that an extraordinary high reliability of AAL technologies is surely one of their crucial points for the acceptance by the users. In order to get deeper insights for the acceptance and therefore, market success of supporting systems for the elderly, further researches regarding the remaining crucial point should be conducted. On the basis of this knowledge more detailed requirements as a part of a high-quality specification of such systems could be made. This would ensure a respectable fundament for the subsequent product engineering.

\section{DATA Limitations}

Several limitations exist with respect to our sample. First, acquiring participants through personal contacts and students might not lead to a representative sample of the German population. Instead, our participants might be younger, more educated, and they might have more interest in and more knowledge of information technology. Second, since our questionnaire consisted of one question each for all non-AALrelated technologies but three questions each for the AALrelated ones, comparisons between these two types of technologies have to be evaluated with care.

Third, due to the usage of a 7-point scale and, thus, a lower limit of $70 \%$ reliability for all technologies, outliers were made impossible. This generates a problem regarding the average expected reliabilities because participants expecting the reliabilities to be below $70 \%$ probably would have chosen the lower limit instead of their true beliefs.

Fourth, a split dividing the sample into two groups with a cut point of 50 years of age does not reveal two groups of equal size. Instead, our sample of "elderly" people consists of 71 persons while the sample of "younger" people consists of nearly twice as much participants $(n=135)$.

\section{FURTHER RESEARCH}

With respect to the expected reliability as well as the acceptance of AAL technologies, further research is necessary to evaluate the success of AAL in the future and - in the long run - to develop possible market entry strategies. The study at hand focuses on the aspect of expected reliabilities and compared AAL to everyday technologies. It made possible a first evaluation of subjective differences between technologies. Additionally, it enabled an analysis of differences between persons of different ages. So general research of age-related effects could be added or developed regarding the issue of AAL.

Further research, nevertheless, is needed to evaluate the influence of reliability on trust and intention to use. Since these two aspects have a significant influence on whether consumers 
buy a product, this can hint producers towards important aspects they have to consider when designing AAL technologies. Furthermore, in a next step an experiment with different reliability levels of AAL technologies will be designed. This differentiation will be used to measure the influence of actual reliability on trust, intention to use, and other variables.

\section{ACKNOWLEDGMENT}

This research was supported by grants from the German Federal Ministry of Education and Research (BMBF). It is part of the project SMILEY (Smart and Independent Living for the Elderly) supported by BMBF under contract 01FC10004.

\section{REFERENCES}

[1] UNDESA, "World population ageing 2009," Department of Economic and Social Affairs: Population Division, New York, 2010.

[2] D. Stroud, "Don't fall for the 50-plus blind spot," Brandweek, vol. 47 (10), 2006.

[3] W. Paulus, J. Hilbert, and W. Potratz, „ICT for housing,” in N. Malanowski, M. Cabrera (Eds.): Information and Communication Technologies for Active Ageing. Opportunities and Challenges for the European Union. IOS Press. Amsterdam, 2009.

[4] BMBF, "Assistenzsysteme im Dienste des älteren Menschen,“ Steckbriefe der ausgewählten Projekte in der BMBF-Fördermaßnahme "Altersgerechte Assistenzsysteme für ein gesundes und unabhängiges Leben - $\quad$ AAL“, $\quad$ URL: http://www.aaldeutschland.de/deutschland/dokumente/projektportrats-aal.pdf (last checked 19.04.2013).

[5] Fraunhofer (2011): „Zuhause Daheim: Das Projekt JUTTA.“ URL: http://www.inhaus.fraunhofer.de/de/Geschaeftsfelder/Health-undCare/jutta.html (last checked 19.04.2013).

[6] W. Heusinger, ,Das intelligente Haus - Entwicklung und Bedeutung für die Lebensqualität, “Frankfurt am Main: Lang, 2005.

[7] S. Solaimani, H. Bouwman, and M. de Reuver, "Smart home: aligning business models and providers processes; a case survey," 21 st Australian Conference on Information Systems Aligning Business Models and Providers Processes, Brisbane, 2010.

[8] J. Botia, A. Villa and J. Palma, "Ambient assisted living system for inhome monitoring of healthy independet elders", Expert Systems with Application, vol. 39, 2012, p. 1.

[9] Commission of the European Communities (2005). Green paper "Confronting demographic change: a new solidarity between the generations". p. 10.
[10] H. Sun, V. de Florio, and N. Gui, "Promise and challenges of Ambient Assisted Living," Sixth International Conference on Information Technology: New Generations, 2009.

[11] D. A. Wiegmann, A. Rich, and H. Zhang, "Automated diagnostic aids: The effects of aid reliability on users' trust and reliance," Theoretical Issues in Ergonomics Science, vol 2 (4), 2001, pp. 352-367.

[12] A. Keller, and S. Rice, "System-wide versus component-specific trust using multiple aids," The Journal of General Psychology, vol. 137 (1), 2010, pp. 114-128.

[13] T. Bahmanziari, J. Pearson, and L. Crosby, "Is trust important in technology adoption? A policy capturing approach," Journal of Computer Information Systems, vol. 43 (4), 2003, pp.46-54.

[14] Oxford Dictionary, URL: http://oxforddictionaries.com/definition/english/reliable, (last checked 19.04.2013).

[15] Oxford Dictionary,

URL: http://oxforddictionaries.com/definition/english/reliable, (last checked 26.04.2013)

[16] E. N. H. Montague, W. W. Winchester, and B. M. Kleiner, "Trust in medical technology by patients and healthcare providers in obstetric work systems," Behaviour \& Information Technology, vol. 29 (5), 2010, pp. 541-554.

[17] R. Parasuraman, and C. Miller, "Trust and etiquette in high-criticality automated systems," Communications of the Association for Computing Machinery, vol 47 (4), 2004, pp. 51-55.

[18] J. Sanchez, G. Calcaterra, and Q. Q. Tran, "Automation in the home: The development of an appropriate system representation and its effects on reliance," Proceedings of the Human Factors and Ergonomics Society $49^{\text {th }}$ annual meeting, 2005.

[19] A. Munoz, E. Serrano, A. Villa, M.Valdes and J. Botia, "An approach for representing sensor data to valdidate alerts in ambient assisted living", Sensors 2012, 2012

[20] M.S. Bartlett, G. Littlewort, I. Fasel., \& J.R. Movellan, „Real time face detection and facial expression recognition: development and application to human computer interaction.", In Proceedings of the Workshop on Computer Vision and Pattern Recognition for Human-Computer Interaction at the 2003 Conference on Computer Vision and Pattern Recognition (pp. 53-58).

[21] F. Steinke, T. Fritsch, D.Brem and S.Simonsen, "Requirement of AAL systems-older perons' trust in sensors and characteristics of AAL technologies," Proceedings of the 5th International Conference on PErvasive Technologies Related to Assistive Environments, Heraklion, Crete, Greece, 2012.

[22] F. Steinke, A. Ingenhoff, and T. Fritsch, "Personal remote support in Ambient Assisted Living - experimental investigation on trust and intention to use of elderly people," unpublished. 